\title{
A HOMOTOPY INVARIANCE THEOREM IN COARSE COHOMOLOGY AND $K$-THEORY
}

\author{
NIGEL HIGSON AND JOHN ROE
}

\begin{abstract}
We introduce a notion of homotopy which is appropriate to the coarse geometry and topology studied by the second author in [7]. We prove the homotopy invariance of coarse cohomology, and of the $K$-theory of the $C^{*}$-algebra associated to a coarse structure on a space. We apply our homotopy invariance results to show that if $M$ is a Hadamard manifold then the inverse of the exponential map at any point 0 induces an isomorphism between the $K$-theory groups of the $C^{*}$-algebras associated to $M$ and its tangent space at 0 (see Theorem 7.9). This result is consistent with a coarse version of the Baum-Connes conjecture.
\end{abstract}

Let $M$ be a complete Riemannian manifold. The second author has introduced in [7] a $C^{*}$-algebra (we shall denote it by $C^{*}(M)$ in this article) whose $K$-theory groups are the receivers for analytic indices of Dirac type operators on $M$. One can ask whether every class in the $K$-theory of $C^{*}(M)$ is the index of some elliptic operator on $M$. The conjecture that this is so, for suitable $M$, is a nonequivariant version of a very well-known conjecture of $P$. Baum and $\mathrm{A}$. Connes in the $K$-theory of group $C^{*}$-algebras. In this paper we shall answer the question affirmatively in the case where $M$ is simply connected and nonpositively curved.

In Section 1 we shall introduce a notion of coarse homotopy appropriate to the coarse geometry introduced in [7]. Our main result, proved in Section 6, asserts that the $K$-theory of $C^{*}(M)$ is a coarse homotopy invariant. The key step in the argument is adapted from G. Kasparov's proof of the homotopy invariance of his analytic $K$-homology groups [5]. We shall also prove the analogous result for the coarse cohomology groups of $M$ (see Section 2), using the basic techniques introduced in [7].

If $M$ is a simply connected, nonpositively curved, complete Riemannian manifold then a simple calculation (performed in Section 7) shows that the inverse of the exponential map at any point 0 in $M$ is a coarse homotopy equivalence. This reduces the calculation of $K_{*}\left(C^{*}(M)\right)$ to the case of the Euclidean space $T_{0} M$, which was worked out in [3].

Received by the editors February 17, 1993 and, in revised form, October 6, 1993.

1991 Mathematics Subject Classification. Primary 19K56.

This research was partially supported by the National Science Foundation (USA) and a European Community Research Grant. Nigel Higson is an Alfred P. Sloan Foundation Research Fellow. 
Our homotopy invariance principle may be applied to more general calculations. These will be given elsewhere [4].

\section{COARSE HOMOTOPY}

We recall from [7] that a proper metric space is one in which closed and bounded sets are compact. For this article we adopt the following terminology:

(1.1) Definition. Let $X$ and $Y$ be proper metric spaces. A coarse map from $X$ to $Y$ is a proper Borel map $f: X \rightarrow Y$ such that for every $R>0$ there exists $S>0$ with

$$
d\left(x, x^{\prime}\right) \leq R \quad \Rightarrow \quad d\left(f(x), f\left(x^{\prime}\right)\right) \leq S .
$$

The composition of two coarse maps is a coarse map, and we obtain the category of coarse spaces. It is the category UBB of [7]. For the greater part of this paper we will in fact be working in the subcategory UBC of proper metric spaces and continuous coarse maps; for our purposes this is not a significant restriction, as will be explained at the end of this section.

(1.2) Definition. Let $X$ and $Y$ be proper metric spaces. A coarse homotopy from $X$ to $Y$ is a continuous and proper map

$$
h: X \times[0,1] \rightarrow Y
$$

such that for every $R>0$ there exists $S>0$ with

$$
d\left(x, x^{\prime}\right) \leq R \Rightarrow d\left(h(x, t), h\left(x^{\prime}, t\right)\right) \leq S, \quad \text { for all } t \in[0,1] .
$$

Two coarse maps $f_{0}, f_{1}: X \rightarrow Y$ are coarsely homotopic if there is a coarse homotopy $h: X \times[0,1] \rightarrow Y$ such that

$$
f_{0}(x)=h(x, 0) \text { and } f_{1}(x)=h(x, 1), \quad \text { for all } x \in X .
$$

A coarse map $f: X \rightarrow Y$ is a coarse homotopy equivalence if there is a coarse map $g: Y \rightarrow X$ such that $f \circ g$ and $g \circ f$ are coarsely homotopic to the identity maps on $Y$ and $X$, respectively.

Coarse homotopy is not the same as the "bornotopy" of [7], Definition 2.5. For example, the spaces $\mathbb{R}$ and $\mathbb{Z}$ are bornotopy equivalent, but not coarsely homotopy equivalent, and the euclidean and hyperbolic planes are coarsely homotopy equivalent (see Section 7) but not bornotopy equivalent.

(1.3) Definition. Let $h: X \times[0,1] \rightarrow Y$ be a coarse homotopy. Equip $X \times$ $[0,1]$ with the metric

$$
d_{h}\left((x, t),\left(x^{\prime}, t^{\prime}\right)\right)=d_{X}\left(x, x^{\prime}\right)+\left|t-t^{\prime}\right|+\sup _{0 \leq s \leq 1} d_{Y}\left(h(x, s t), h\left(x^{\prime}, s t^{\prime}\right)\right) .
$$

We shall write $X \times_{h}[0,1]$ in place of $X \times[0,1]$ to indicate that this particular metric has been chosen.

It is clear that $h: X \times_{h}[0,1] \rightarrow Y$ is a coarse map, as is the projection map

$$
\pi: X \times_{h}[0,1] \rightarrow X .
$$

Thanks to the definition of coarse homotopy, so are the maps

$$
e^{t}: X \rightarrow X \times_{h}[0,1] \quad(t \in[0,1]),
$$

defined by $e^{t}(x)=(x, t)$. 
(1.4) Lemma. The projection map $\pi: X \times_{h}[0,1] \rightarrow X$ is a coarse homotopy equivalence.

Proof. We shall show that $e^{0}: X \rightarrow X \times_{h}[0,1]$ is a coarse homotopy inverse. The composition $\pi \circ e^{0}: X \rightarrow X$ is the identity map. The composition

$$
e^{0} \circ \pi: X \times_{h}[0,1] \rightarrow X \times_{h}[0,1]
$$

is homotopic to the identity via the coarse homotopy

$$
\tilde{h}((x, t), s)=(x, s t) \text {. }
$$

(1.5) Lemma. Let $F$ be a functor on the category of coarse spaces. The following are equivalent:

(1) $F$ is coarse homotopy invariant, meaning that

$$
F\left(f_{0}\right)=F\left(f_{1}\right): F(X) \rightarrow F(Y),
$$

whenever $f_{0}, f_{1}: X \rightarrow Y$ are coarsely homotopic maps.

(2) For every coarse homotopy $h: X \times[0,1] \rightarrow Y$ we have

$$
F\left(e^{0}\right)=F\left(e^{1}\right): F(X) \rightarrow F\left(X \times_{h}[0,1]\right) .
$$

(3) For every coarse homotopy $h: X \times[0,1] \rightarrow Y$ the morphism

$$
F(\pi): F\left(X \times_{h}[0,1]\right) \rightarrow F(X)
$$

is an isomorphism.

Proof. Clear.

Some important examples of coarse homotopies are given in Section 7.

Remark. In order to have a notion of equivalence that includes both coarse homotopy and bornotopy, we may relax Definition 1.2 as follows. A generalized coarse homotopy is a map $h: X \times[0,1] \rightarrow Y$, satisfying the estimate in Definition 1.2, but which is required only to be pseudocontinuous and not necessarily continuous. This means that there is some constant $T>0$ such that, for all $\xi \in X \times[0,1], \xi$ is an interior point of $f^{-1}(B(f(\xi) ; T))$. It is easy to check that both coarse homotopies and bornotopies are generalized coarse homotopies.

One can show that if $Y$ is a path metric space and $h: X \times[0,1] \rightarrow Y$ is a generalized coarse homotopy, then there exist another path metric space $Y^{\prime}$, a bornotopy-equivalence $i: Y \rightarrow Y^{\prime}$, and a coarse homotopy $h^{\prime}: X \times[0,1] \rightarrow Y^{\prime}$ such that the obvious diagram is bornotopy commutative (see [8, Section 3]). Thus any functor $F$ which is both coarse homotopy invariant and bornotopy invariant will be invariant under generalized coarse homotopies, at least on the subcategory of path spaces.

For the remainder of this paper all coarse maps and homotopies are assumed to be continuous.

\section{HOMOTOPY INVARIANCE OF COARSE COHOMOLOGY}

Let $Y$ be a proper metric space and form the coarse cohomology $H X^{*}(Y)$ as in [7]. It is contravariantly functorial on the category of coarse spaces. Our objective in this section is to prove the following result. 
(2.1) Theorem. If $f_{0}, f_{1}: X \rightarrow Y$ are coarsely homotopic then

$$
f_{0}^{*}=f_{1}^{*}: H X^{*}(Y) \rightarrow H X^{*}(X)
$$

We recall some definitions from [7]. A good cover of $Y$ is a locally finite open cover by relatively compact sets. An anti-Čech system on $Y$ is a sequence $\left\{\mathscr{U}_{n}\right\}$ of good covers on $Y$ for which there exists a sequence of real numbers $R_{n} \rightarrow \infty$ such that:

(1) the diameter of each member of $\mathscr{U}_{n}$ is no more than $R_{n}$; and

(2) each subset of $X$ of diameter $R_{n-1}$ or less is contained in some member of $\mathscr{U}_{n}$.

If $\left\{\mathscr{U}_{n}\right\}$ is an anti-Čech system then each $\mathscr{U}_{n}$ is a refinement of $\mathscr{U}_{n+1}$. Forming the Čech cohomology with compact supports $H_{c}^{*}(\mathscr{U} ; \mathbb{R})$ we obtain an inverse system $^{1}$

$$
H_{c}^{*}\left(\mathscr{U}_{1}\right) \leftarrow H_{c}^{*}\left(\mathscr{U}_{2}\right) \leftarrow H_{c}^{*}\left(\mathscr{U}_{3}\right) \leftarrow \cdots
$$

Suppose that $\left\{\mathscr{U}_{j_{n}}\right\}$ is a subsequence of $\left\{\mathscr{U}_{n}\right\}$. It is an anti-Čech system in its own right. We make note of the following fact.

Lemma. The commuting diagram

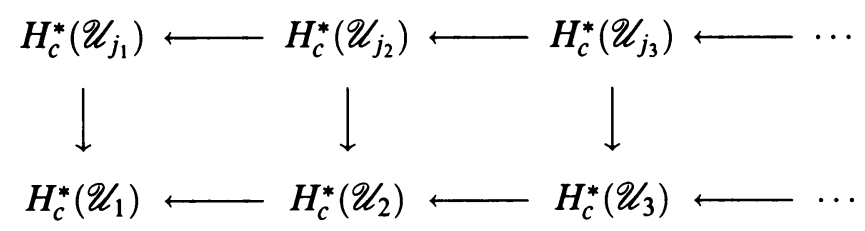

give rise to isomorphisms

$$
\underset{\lim }{\longleftarrow} H_{c}^{q}\left(\mathscr{U}_{j_{n}} ; \mathbb{R}\right) \cong \lim H_{c}^{q}\left(\mathscr{U}_{n} ; \mathbb{R}\right), \quad \lim ^{1} H_{c}^{q}\left(\mathscr{U}_{j_{n}} ; \mathbb{R}\right) \cong \lim ^{1} H_{c}^{q}\left(\mathscr{U}_{n} ; \mathbb{R}\right) .
$$

(As usual, $\lim ^{1}$ denotes the derived functor of $\lim _{\longleftarrow}$.)

We now recall an important result which reduces many questions about $H X^{*}(Y)$ to problems in Čech theory.

(2.3) Theorem. (See [7, Theorem 3.14].) Let $\left\{\mathscr{U}_{n}\right\}$ be an anti-Čech system on $Y$. There is a short exact sequence

$$
0 \longrightarrow \lim ^{1} H_{c}^{q-1}\left(\mathscr{U}_{n} ; \mathbb{R}\right) \rightarrow H X^{q}(Y) \rightarrow \underset{\lim }{\longleftarrow} H_{c}^{q}\left(\mathscr{U}_{n} ; \mathbb{R}\right) \rightarrow 0
$$

It is natural, in the sense that if $f: X \rightarrow Y$ is a coarse map, and if $\left\{\mathscr{V}_{n}\right\}$ is any anti-Čech system on $X$ which refines $\left\{f^{*} \mathscr{U}_{n}\right\}$, then the diagram

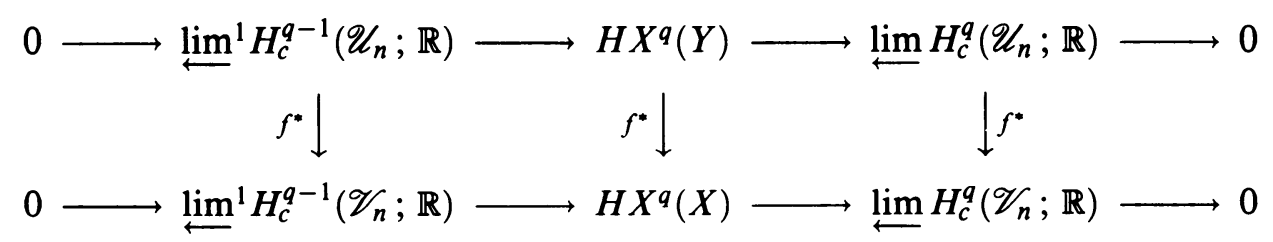

commutes.

\footnotetext{
${ }^{1}$ Strictly speaking, here and below we must choose refining maps to obtain induced maps on cohomology.
} 


\section{(2.4) Lemma.}

(a) Let $h: X \times[0,1] \rightarrow Y$ be a coarse homotopy and let $f_{t}(x)=h(x, t)$. Let $\mathscr{U}$ be any good cover of $Y$ and let $\mathscr{V}$ be any good cover of $X$ which refines $f_{t}^{*} \mathscr{U}$ for all $t$. Then

$$
f_{0}^{*}=f_{1}^{*}: H_{c}^{*}(\mathscr{V} ; \mathbb{R}) \rightarrow H_{c}^{*}(\mathscr{U} ; \mathbb{R}) .
$$

(b) Let $h$ and $f_{t}$ be as in part (a). Let $\left\{\mathscr{U}_{n}\right\}$ be an anti-Čech system on $Y$ and let $\left\{\mathscr{V}_{n}\right\}$ be an anti-Čech system on $X$. There is a subsequence $\left\{\mathscr{U}_{j_{n}}\right\}$ such that $\left\{\mathscr{V}_{n}\right\}$ is a refinement of $\left\{f_{t}^{*} \mathscr{U}_{j_{n}}\right\}$, for all $t$.

Proof. Part (a) is Lemma 3.12 in [7]. For part (b), according to Definition 1.2, for every $R>0$ there is some $S>0$ such that if $V$ is any set of diameter $R$ or less then for all $t$, the set $f_{t}[V]$ has diameter $S$ or less. So for each fixed $n$ the sets $f_{t}[V]\left(t \in[0,1]\right.$ and $\left.V \in \mathscr{V}_{n}\right)$, have uniformly bounded diameter. Consequently they are all contained within the members of some $\mathscr{U}_{j_{n}}$.

(2.5) Lemma. Let $f: X \rightarrow Y$ be a coarse homotopy equivalence. There is an anti-Čech system $\left\{\mathscr{U}_{n}\right\}$ on $Y$ and an anti-Čech system $\left\{\mathscr{V}_{n}\right\}$ on $X$, which refines $\left\{f^{*} \mathscr{U}_{n}\right\}$, such that the induced maps

$$
f^{*}: \lim _{\longleftarrow} H_{c}^{q}\left(\mathscr{U}_{n} ; \mathbb{R}\right) \rightarrow \lim _{\longleftarrow} H_{c}^{q}\left(\mathscr{V}_{n} ; \mathbb{R}\right)
$$

and

$$
f^{*}: \lim ^{1} H_{c}^{q}\left(\mathscr{U}_{n} ; \mathbb{R}\right) \rightarrow \lim ^{1} H_{c}^{q}\left(\mathscr{V}_{n} ; \mathbb{R}\right)
$$

are isomorphisms.

Proof. This follows from Lemmas 2.2 and 2.4.

Proof of Theorem 2.1. By Lemma 1.5 it is enough to show that the map

$$
\pi^{*}: H X^{q}(X) \rightarrow H X^{q}\left(X \times_{h}[0,1]\right)
$$

is an isomorphism. Using Theorem 2.2, along with the Five Lemma, the proof follows from (2.5).

\section{The $C^{*}$-Algebra of A COARSE SPACE}

We review some ideas developed in [3] and [7].

(3.1) Definition. Let $X$ be a proper metric space. An $X$-module is a Hilbert space $\mathscr{H}$ equipped with a faithful and nondegenerate representation of the $C^{*}$-algebra $C_{0}(X)$ whose image contains no nonzero compact operator.

Our definition of $X$-module corresponds to the notion of "standard $X$ module" in [7, Definition 4.2], except that we have included the extra condition of nondegeneracy.

(3.2) Definition. Let $\mathscr{H}_{X}$ and $\mathscr{H}_{Y}$ be $X$ and $Y$-modules, respectively, and let $T: \mathscr{H}_{X} \rightarrow \mathscr{H}_{Y}$ be a bounded linear operator. The support of $T$, denoted $\operatorname{Supp}(T)$, is the complement of the set of all points $(x, y) \in X \times Y$ for which 
there exist continuous, compactly supported functions $\phi$ and $\psi$ on $X$ and $Y$, respectively, with:

$$
\psi T \phi=0, \quad \phi(x) \neq 0, \quad \text { and } \quad \psi(y) \neq 0 .
$$

For example, if $T$ is an integral operator

$$
T f(y)=\int_{X} k(y, x) f(x) d x
$$

(mapping $L^{2}(X)$ to $L^{2}(Y)$ ), then $\operatorname{Supp}(T)=\operatorname{Supp}(k)$.

(3.3) Definition. Let $\mathscr{H}_{X}$ be an $X$-module. The propagation of a bounded linear operator $T: \mathscr{H}_{X} \rightarrow \mathscr{H}_{X}$ is the quantity

$$
\operatorname{Prop}(T)=\sup \{d(x, y) \mid(x, y) \in \operatorname{Supp}(T)\} .
$$

We say that $T$ has finite propagation if $\operatorname{Prop}(T)<\infty$.

(3.4) Definition. A bounded operator $T: \mathscr{H}_{X} \rightarrow \mathscr{H}_{X}$ is locally compact if $\psi T$ and $T \psi$ are compact operators, for every $\psi \in C_{0}(X)$.

(3.5) Definition. The set of locally compact, finite propagation operators on $\mathscr{H}_{X}$ is a $*$-subalgebra of the algebra of all bounded operators on $\mathscr{H}_{X}$. Denote by $C^{*}\left(X, \mathscr{H}_{X}\right)$ the $C^{*}$-algebra obtained by closing this *-subalgebra in the operator norm.

This is the same as the $C^{*}$-algebra $\overline{\mathscr{B}}_{\mathscr{H}}$ in [7, Lemma 4.12].

If $X$ is a complete Riemannian manifold and $D$ is a Dirac-type operator on $X$, then for every $f \in C_{0}(\mathbb{R})$ the operator $f(D)$ lies in $C^{*}\left(X, \mathscr{H}_{X}\right.$ ) (where $\mathscr{H}_{X}$ is the Hilbert space of $L^{2}$-spinors on $\left.X\right)$. It follows that $D$ possesses an "index" in the $K$-theory of $C^{*}\left(X, \mathscr{H}_{X}\right)$ (see [7]), which explains our interest in this $C^{*}$-algebra.

We shall need the following three lemmas in the coming sections.

(3.6) Lemma. Every finite propagation operator $T: \mathscr{H}_{X} \rightarrow \mathscr{H}_{X}$ is a multiplier of $C^{*}\left(X, \mathscr{H}_{X}\right)$.

Proof. Suppose that $S$ is a locally compact, finite propagation operator on $\mathscr{H}_{X}$. The compositions $T S$ and $S T$ have finite propagation (at most the sum of the propagations of $S$ and $T$ ). We must show that $S T$ and $T S$ are locally compact. If $\phi \in C_{c}(X)$ then there is some $\phi^{\prime} \in C_{c}(X)$ such that $\phi T \phi^{\prime}=\phi T$. (It suffices to choose $\phi^{\prime}$ so that $\phi^{\prime} \equiv 1$ within a distance $\operatorname{Prop}(T)$ of $\operatorname{Supp}(\phi)$.) Then

$$
\phi T S=\phi T \cdot \phi^{\prime} S,
$$

which, being a product of a bounded and a compact operator, is compact. Obviously $T S \phi$ is compact since $S \phi$ is. This shows that $T S$ is locally compact. The case of $S T$ is similar.

(3.7) Lemma. Let $\phi \in C_{0}(X)$ and let

$$
\operatorname{Lip}(\phi)=\inf \left\{K:\left|\phi(x)-\phi\left(x^{\prime}\right)\right| \leq K d\left(x, x^{\prime}\right) \text {, for all } x, x^{\prime} \in X\right\} .
$$

Then for any bounded operator $T: \mathscr{H}_{X} \rightarrow \mathscr{H}_{X}$,

$$
\|T \phi-\phi T\| \leq \text { constant } \cdot\|T\| \cdot \operatorname{Prop}(T) \cdot \operatorname{Lip}(\phi) .
$$


Proof. See Proposition 5.18 of [7].

(3.8) Lemma. The $C^{*}$-algebra $C^{*}\left(X, \mathscr{H}_{X}\right)$ has an approximate identity consisting of projections.

Proof. By Spectral Theory the representation of $C_{0}(X)$ on $\mathscr{H}_{X}$ extends to a representation on $\mathscr{H}_{X}$ of the algebra of bounded Borel functions on $X$. Partition $X$ into uniformly bounded Borel pieces $X_{j}$ in such a way that each bounded set in $X$ intersects only finitely many of the $X_{j}$. There is a corresponding decomposition $\mathscr{H}_{X}=\bigoplus_{j} \mathscr{H}_{X_{j}}$, and the operators formed by taking direct sums of finite rank projection operators on each $\mathscr{X}_{X_{j}}$ constitute an approximate identity for $C^{*}\left(X, \mathscr{H}_{X}\right)$.

As already indicated, we are interested in the groups $K_{*}\left(C^{*}\left(X, \mathscr{H}_{X}\right)\right)$. They may be made functorial on the coarse category in the following way.

(3.9) Proposition. Let $\mathscr{H}_{X}$ and $\mathscr{H}_{Y}$ be $X$ and $Y$-modules, respectively, and let $f: X \rightarrow Y$ be a coarse map. There is an isometry of Hilbert spaces $V: \mathscr{H}_{X} \rightarrow \mathscr{H}_{Y}$ such that for some $R>0$,

$$
\operatorname{Supp}(V) \subseteq\{(x, y) \in X \times Y: d(f(x), y) \leq R\} .
$$

We have that

$$
V C^{*}\left(X, \mathscr{H}_{X}\right) V^{*} \subset C^{*}\left(Y, \mathscr{H}_{Y}\right),
$$

and the induced map on $K$-theory,

$$
\operatorname{Ad}(V)_{*}: K_{*}\left(C^{*}\left(X, \mathscr{H}_{X}\right)\right) \rightarrow K_{*}\left(C^{*}\left(Y, \mathscr{H}_{Y}\right)\right),
$$

is independent of the choice of $V$.

Proof. See Section 4 of [3].

We shall denote the map $\operatorname{Ad}(V)_{*}$ in Proposition 3.9 by $f_{*}$.

It follows from the proposition that $K_{*}\left(C^{*}\left(X, \mathscr{H}_{X}\right)\right)$ does not depend on the choice of $\mathscr{H}_{X}$, up to canonical isomorphism (in fact, up to noncanonical isomorphism, the $C^{*}$-algebra itself does not depend on the choice of $\mathscr{H}_{X}$ ). Associating to each proper metric space $X$ a module $\mathscr{H}_{X}$ we obtain a functor on the coarse category.

\section{SOME REMARKS ON $K$-THEORY}

Let $B$ be a $C^{*}$-algebra and let $M(B)$ be the multiplier algebra of $B$ [6]. Let

$$
D(B)=\left\{\left(d_{1}, d_{2}\right) \in M(B) \times M(B): d_{1}-d_{2} \in B\right\} .
$$

This $C^{*}$-algebra fits into a short exact sequence

$$
0 \rightarrow B \stackrel{j}{\longrightarrow} D(B) \stackrel{\pi}{\longrightarrow} M(B) \rightarrow 0,
$$

where $\pi$ is the projection onto the second factor and $j(b)=(b, 0)$. The sequence is split by the $*$-homomorphism

$$
s: M(B) \rightarrow D(B)
$$


given by the formula $s(d)=(d, d)$, and so it gives rise to the following split short exact sequence of $K$-theory groups:

$$
0 \longrightarrow K_{*}(B) \stackrel{j_{*}}{\longrightarrow} K_{*}(D(B)) \stackrel{\pi_{*}}{\longrightarrow} K_{*}(M(B)) \longrightarrow 0 .
$$

(4.3) Definition. Let $e, f$ be a pair of idempotents in $M(B)$ (that is, $e^{2}=e$ and $f^{2}=f$ ) such that

$$
e-f \in B \text {. }
$$

In other words, let $(e, f)$ be an idempotent in $D(B)$. We define a class

$$
[e] \ominus[f] \in K_{0}(B)
$$

by the requirement that

$$
j_{*}([e] \ominus[f])=[(e, e)]-[(f, e)]
$$

in $K_{0}(D(B))$.

(4.4) Lemma.

(a) If $(e, f)$ is an idempotent in $D(B)$ and $e \geq f$, meaning that $e f=f e=$ $f$, then $e-f$ is an idempotent in $B$ and

$$
[e] \ominus[f]=[e-f] .
$$

(b) If $\left(e_{t}, f_{t}\right) \quad(0 \leq t \leq 1)$ is a norm continuous family of idempotents in $D(B)$ then

$$
\left[e_{0}\right] \ominus\left[f_{0}\right]=\left[e_{1}\right] \ominus\left[f_{1}\right] .
$$

Proof. Straightforward.

We shall be interested in idempotents in $D(B)$ which are constructed as follows. Fix an idempotent $e$ in $M(B)$. Let $p$ and $q$ be idempotents in $M(B)$ such that

$$
\|e p-p e\|<\frac{1}{4\|p\|\|e\|}
$$

and

$$
\|e q-q e\|<\frac{1}{4\|q\|\|e\|},
$$

and also

$$
e(p-q) \in B \text {. }
$$

The estimates imply that

$$
\left\|(e p)^{2}-e p\right\|<\frac{1}{4} \text { and }\left\|(e q)^{2}-e q\right\|<\frac{1}{4},
$$

which in turn implies that the spectra of $e p$ and $e q$ are each disjoint from the line $\operatorname{Re}(z)=1 / 2$ in the complex plane $\mathbb{C}$. Let $\gamma$ be any contour in $\mathbb{C}$ surrounding the components of $\operatorname{Spec}(e p)$ and $\operatorname{Spec}(e q)$ to the right of this line. As is well known, the elements

$$
\overline{e p}=\frac{1}{2 \pi i} \int_{\gamma}(e p-\lambda)^{-1} d \lambda
$$


and

$$
\overline{e q}=\frac{1}{2 \pi i} \int_{\gamma}(e q-\lambda)^{-1} d \lambda
$$

are then idempotents in $M(B)$. It follows from (4.5c) that $\overline{e p}-\overline{e q} \in B$, and so we can define a class

$$
[\overline{e q}] \ominus[\overline{e p}] \in K_{0}(B) .
$$

\section{(4.6) Lemma.}

(a) The class $[\overline{e q}] \ominus[\overline{e p}] \in K_{0}(B)$ depends only on the norm homotopy class of the pair $p, q$ satisfying (4.5).

(b) If $e$ commutes with $p$ and $q$ then

$$
[\overline{e q}] \ominus[\overline{e p}]=[e q] \ominus[e p] \in K_{0}(B),
$$

and if in addition $q \geq p$ then

$$
[\overline{e q}] \ominus[\overline{e p}]=[e(q-p)] \in K_{0}(B)
$$

Proof. Part (a) follows from (4.4b). Part (b) follows from (4.4a), together with the observation that if $e$ commutes with $p$ and $q$ then the elements $e p$ and $e q$ are already idempotents, so that $\overline{e p}=e p$ and $\overline{e q}=e q$.

Finally, it will simplify our arguments to make note of the following remarks concerning generators for $K_{0}$ and $K_{1}$.

(4.7) Lemma.

(a) Suppose that a $C^{*}$-algebra $B$ has an approximate identity consisting of projections. Then $K_{0}(B)$ is generated by the $K$-theory classes of projections in matrix algebras over $B$ (so it is not necessary to adjoin a unit to $B$ ).

(b) Suppose, in addition that for every $n$ there is a *-homomorphism $M_{n}(B)$ $\rightarrow B$ whose composition with the standard inclusion $B \rightarrow M_{n}(B)$ induces the identity map on the $K$-theory of $B$. Then $K_{0}(B)$ is generated by equivalence classes of projections in $B$ (it is not necessary to pass to matrices over $B$ ).

Denote by $C\left(S^{1}\right)$ the $C^{*}$-algebra of continuous functions on the circle. For any $C^{*}$-algebra $A$ there is a natural isomorphism

$$
K_{0}\left(C\left(S^{1}\right) \otimes A\right) \cong K_{0}(A) \oplus K_{1}(A) .
$$

(4.8) Lemma. The group

$$
K_{0}\left(C\left(S^{1}\right) \otimes C^{*}\left(X, \mathscr{H}_{X}\right)\right)
$$

is generated by the $K$-theory classes of projections in $C\left(S^{1}\right) \otimes C^{*}\left(X, \mathscr{H}_{X}\right)$.

Proof. It follows from Lemma 3.8 that $C^{*}\left(X, \mathscr{H}_{X}\right)$, and hence $C\left(S^{1}\right) \otimes C^{*}(X)$, has an approximate identity consisting of projections. Since

$$
M_{n}\left(C\left(S^{1}\right) \otimes C^{*}\left(X, \mathscr{H}_{X}\right)\right) \cong C\left(S^{1}\right) \otimes C^{*}(X, \underbrace{\mathscr{H}_{X} \oplus \cdots \oplus \mathscr{H}_{X}}_{n \text { times }})
$$

it follows from Proposition 3.9 that the hypothesis in $(4.7 \mathrm{~b})$ is satisfied for $B=C\left(S^{1}\right) \otimes C^{*}\left(X, \mathscr{H}_{X}\right)$.

\section{COARSE HOMOTOPY INVARIANCE IN $K$-THEORY}

In this section and the next we shall omit the module $\mathscr{H}_{X}$ from our notation, writing $C^{*}(X)$ in place of $C^{*}\left(X, \mathscr{X}_{X}\right)$. Our objective is the following result. 
(5.1) Theorem. If $f^{0}, f^{1}: X \rightarrow Y$ are coarsely homotopic coarse maps then

$$
f_{*}^{0}=f_{*}^{1}: K_{*}\left(C^{*}(X)\right) \rightarrow K_{*}\left(C^{*}(Y)\right) \text {. }
$$
that

$$
e_{*}^{0}=e_{*}^{1}: K_{0}\left(C\left(S^{1}\right) \otimes C^{*}(X)\right) \rightarrow K_{0}\left(C\left(S^{1}\right) \otimes C^{*}\left(X \times_{h}[0,1]\right)\right) .
$$

In this section we will lay the groundwork for the proof by giving a general procedure for mapping elements ${ }^{2}$ of $K_{0}\left(C\left(S^{1}\right) \otimes C^{*}(X)\right)$ to elements of the group $K_{0}\left(C\left(S^{1}\right) \otimes C^{*}\left(X \times_{h}[0,1]\right)\right)$, using as auxiliary data suitable families of operators on $L^{2}(\mathbb{R})$. In the following section we will exhibit such families which implement a 'homotopy' between $e_{*}^{0}$ and $e_{*}^{1}$, following an argument given by Kasparov [5] to establish the homotopy invariance of $K$-homology.

Choose a fully supported Borel measure on $X$ and form the Hilbert space $L^{2}(X)$. As long as $X$ has no isolated points this is an $X$-module. (If there are isolated points we should take an infinite direct sum of copies of $L^{2}(X)$, but in order to keep things simple we shall not mention this point further.) We shall take $L^{2}(X)$ as the space on which $C^{*}(X)$ acts:

$$
\mathscr{H}_{X}=L^{2}(X) \text {. }
$$

Equip $\mathbb{R}$ with Lebesgue measure and form the Hilbert space $L^{2}(\mathbb{R})$. If $\phi$ is a continuous function on $[0,1]$ then extend it to a continuous function $\tilde{\phi}$ on $\mathbb{R}$ by

$$
\tilde{\phi}(t)= \begin{cases}\phi(0) & \text { if } t \leq 0, \\ \phi(t) & \text { if } 0 \leq t \leq 1, \\ \phi(1) & \text { if } t \geq 1\end{cases}
$$

View $\phi$ as acting on $L^{2}(\mathbb{R})$ via pointwise multiplication by $\tilde{\phi}$. In this way $L^{2}(\mathbb{R})$ becomes a $[0,1]$-module.

The tensor product $L^{2}(X) \otimes L^{2}(\mathbb{R}) \cong L^{2}(X \times \mathbb{R})$ becomes an $X \times_{h}[0,1]$ module, in the evident manner. We shall take the direct sum of two copies of this space as the module on which $C^{*}\left(X \times_{h}[0,1]\right)$ acts:

$$
\mathscr{H}_{X \times_{h}[0,1]}=L^{2}(X \times \mathbb{R}) \oplus L^{2}(X \times \mathbb{R}) .
$$

Choose norm-one functions

$$
\psi_{0} \in L^{2}(-\infty, 0) \subset L^{2}(\mathbb{R}) \text { and } \psi_{1} \in L^{2}(1, \infty) \subset L^{2}(\mathbb{R}),
$$

and define isometries

$$
V_{0}, V_{1}: L^{2}(X) \rightarrow L^{2}(X \times \mathbb{R}) \oplus L^{2}(X \times \mathbb{R})
$$

by

$$
V_{0} \phi=\left(0, \phi \otimes \psi_{0}\right) \quad \text { and } \quad V_{1} \phi=\left(0, \phi \otimes \psi_{1}\right) \quad\left(\phi \in L^{2}(X)\right) .
$$

Then, according to Proposition 3.9, $e_{*}^{i}=\operatorname{Ad}\left(V_{i}\right)_{*}(i=0,1)$. We note that

$$
\operatorname{Ad}\left(V_{i}\right)(T)=\left(\begin{array}{cc}
0 & 0 \\
0 & T \otimes p_{i}
\end{array}\right) \quad(i=0,1),
$$

where $p_{0}$ and $p_{1}$ are projection operators onto the one-dimensional subspaces of $L^{2}(\mathbb{R})$ spanned by $\psi_{0}$ and $\psi_{1}$, respectively.

\footnotetext{
${ }^{2}$ One might expect this procedure to define a homomorphism, but this does not seem to be immediate from our argument and is not required by it.
} 
(5.4) Definition. Let $\varepsilon>0$. An $\varepsilon$-flat partition of unity $\sigma$ on $X$ is a partition of unity $\sigma_{1}, \sigma_{2}, \ldots$ on $X$ consisting of compactly supported functions such that:

(5.4a) $\sigma_{i} \sigma_{j}=0$ if $|i-j| \geq 2$; and

(5.4b) $\left|\sigma_{i}(x)-\sigma_{i}\left(x^{\prime}\right)\right| \leq \varepsilon 2^{-i} d\left(x, x^{\prime}\right)$ for all $i$, and all $x, x^{\prime} \in X$.

Such a partition of unity may for example be defined by starting with a partition of unity $s_{1}, s_{2}, \ldots$ on $[0, \infty)$ with the above properties, and then forming $\sigma_{i}(x)=s_{i}(d(0, x))$, where 0 is some fixed point in $X$.

We shall be interested in operators on $L^{2}\left(X \times_{h}[0,1]\right)$ of the form

$$
F=\sum_{i} \sigma_{i} \otimes F_{i},
$$

where $\sigma$ is an $\varepsilon$-flat partition of unity and $F_{1}, F_{2}, \ldots$ are operators on $L^{2}(\mathbb{R})$ with the following properties:

(5.6a) $\sup _{i}\left\|F_{i}\right\|<\infty$;

(5.6b) the operators $F_{i}$ and $F_{j}$ are compact perturbations of one another, for all $i$ and $j$

(5.6c) the operators $F_{i} F_{i}^{*}-1$ and $F_{i}^{*} F_{i}-1$ are compact, for all $i$; and

(5.6d) viewing $L^{2}(\mathbb{R})$ as a $[0,1]$-module as above, the propagation of $F_{i}$ is so small that

$$
\left|t-t^{\prime}\right|<\operatorname{Prop}\left(F_{i}\right) \Rightarrow d_{h}\left((x, t),\left(x, t^{\prime}\right)\right) \leq 1, \quad \text { for all } x \in \operatorname{Supp}\left(\sigma_{i}\right) \text {. }
$$

Such a family $\mathbf{F}=\left(F_{i}\right)$ of operators will be referred to as a good family, and the set of such familes will be topologized by the supremum norm. The operator $F$ defined by (5.5) will be called the sum of the good family $\mathbf{F}$ (with respect to $\sigma$ ), and may be denoted by $\sigma \cdot \mathbf{F}$. It follows from (5.4a) and (5.6a) that the sum (5.5) does converge in the strong operator topology, and

$$
\|\sigma \cdot \mathbf{F}\| \leq \text { constant } \cdot \sup _{i}\left\|F_{i}\right\|
$$

where the constant does not depend on the choice of $\sigma$. Thus, the map $\mathbf{F} \mapsto \sigma \cdot \mathbf{F}$ is continuous.

(5.7) Lemma. Let $F=\sigma \cdot \mathbf{F}$ be the sum of a good family. Then, viewing $L^{2}(X \times \mathbb{R})$ as an $X \times_{h}[0,1]$-module, the operator $F$ has finite propagation.

Proof. Viewing $L^{2}(X \times \mathbb{R})$ as an $X \times_{h}[0,1]$-module, it follows from the last condition that the operator $\sigma_{i} \otimes F_{i}$ has propagation at most one. Since the support of $\sum_{i} \sigma_{i} \otimes F_{t_{i}}$ is contained within the closure of the union of the supports of the $\sigma_{i} \otimes F_{t_{i}}$, the lemma follows from this.

Given $F$ as above, form the operator

$$
R=\left(\begin{array}{cc}
\left(2-F F^{*}\right) F & F F^{*}-1 \\
1-F^{*} F & F^{*}
\end{array}\right)
$$

on $L^{2}(X \times \mathbb{R}) \oplus L^{2}(X \times \mathbb{R})$. It is an invertible operator, with inverse

$$
R^{-1}=\left(\begin{array}{cc}
F^{*} & 1-F^{*} F \\
F F^{*}-1 & \left(2-F F^{*}\right) F
\end{array}\right) .
$$


These formulas are taken from [2]. Let $Q$ be the projection

$$
Q=\left(\begin{array}{ll}
1 & 0 \\
0 & 0
\end{array}\right),
$$

on $L^{2}(X \times \mathbb{R}) \oplus L^{2}(X \times \mathbb{R})$ and form the idempotent

$$
P=R Q R^{-1} \text {. }
$$

We note that all these operators depend continuously on the initial choice of good family $\mathbf{F}$. When we want to make this choice explicit we will write $P$ as $P(\sigma \cdot \mathbf{F})$, and so on.

(5.10) Lemma. The operators $R, R^{-1}$ and $P$ have finite propagation, and hence are multipliers of $C^{*}\left(X \times_{h}[0,1]\right)$.

Proof. This is a consequence of Lemma 5.7.

We begin the construction of certain classes in $K_{0}\left(C\left(S^{1}\right) \otimes C^{*}\left(X \times_{h}[0,1]\right)\right)$. View $C\left(S^{1}\right) \otimes C^{*}(X)$ as the algebra of continuous functions from the circle into $C^{*}(X)$, and view $C\left(S^{1}\right) \otimes C^{*}\left(X \times_{h}[0,1]\right)$ similarly. For each $e \in C\left(S^{1}\right) \otimes$ $C^{*}(X)$ let

$$
E=\left(\begin{array}{cc}
e \otimes 1 & 0 \\
0 & e \otimes 1
\end{array}\right)
$$

It is a norm-continuous function on the circle with values in the bounded operators on $L^{2}(X \times \mathbb{R}) \oplus L^{2}(X \times \mathbb{R})$.

\section{(5.11) Lemma.}

(a) $E$ is a multiplier of $C\left(S^{1}\right) \otimes C^{*}\left(X \times_{h}[0,1]\right)$.

(b) Fix an $\varepsilon$-flat partition of unity $\sigma$ on $X$ and view the projections $Q$ and $P=P(\sigma \cdot \mathbf{F})$ defined by (5.8) (5.9) as constant functions from the circle into the bounded operators on $\mathscr{H}_{X \times{ }_{h}[0,1]}$. Then

$$
E(P-Q) \in C\left(S^{1}\right) \otimes C^{*}\left(X \times_{h}[0,1]\right) .
$$

(c) Let $e \in C\left(S^{1}\right) \otimes C^{*}(X)$, let $\delta>0$, and let $\mathbb{F}$ be a bounded set of good families. There is a constant $\varepsilon>0$ such that

$$
\|E P(\sigma \cdot \mathbf{F})-P(\sigma \cdot \mathbf{F}) E\|<\delta \quad \forall \mathbf{F} \in \mathbb{F},
$$

for every $\varepsilon$-flat partition of unity $\sigma$ on $X$.

Proof. Suppose first that $e$ has finite propagation, meaning that $e$ takes values in the dense subalgebra of $C^{*}(X)$ consisting of the locally compact operators with finite propagation, with the propagation uniformly bounded on $S^{1}$. Such functions $e$ constitute a dense subalgebra of $C\left(S^{1}\right) \otimes C^{*}(X)$. It follows from the definition of the metric $d_{h}$ on $X \times_{h}[0,1]$, and the definition of coarse homotopy, that $e \otimes 1$ has finite propagation as well. This observation and an approximation argument prove (a).

It suffices to prove (b) for operators $e$ with finite propagation. It follows from Lemma 5.10 that $E(P-Q)$ has finite propagation, so it suffices to prove that it is locally compact (i.e. that it takes values in the locally compact operators on $\left.\mathscr{H}_{X \times_{h}[0,1]}\right)$. Since

$$
E(P-Q)=\left(\begin{array}{cc}
e \otimes 1 & 0 \\
0 & e \otimes 1
\end{array}\right)\left(\begin{array}{cc}
0 & 1-F F^{*} \\
1-F^{*} F & 0
\end{array}\right) R^{-1}
$$


it suffices to show that for any $\psi \in C_{c}(X)$ the functions

$$
\begin{array}{ll}
(\psi \otimes 1)(e \otimes 1)\left(1-F F^{*}\right), & (e \otimes 1)\left(1-F F^{*}\right)(\psi \otimes 1), \\
(\psi \otimes 1)(e \otimes 1)\left(1-F^{*} F\right), & \text { and } \quad(e \otimes 1)\left(1-F^{*} F\right)(\psi \otimes 1)
\end{array}
$$

on $L^{2}(X \times \mathbb{R})$ are compact-operator valued. Since $e$ has finite propagation there is some $\psi^{\prime} \in C_{c}(X)$ with $\psi e \psi^{\prime}=\psi e$. Then

$$
(\psi \otimes 1)(e \otimes 1)\left(1-F F^{*}\right)=(\psi e \otimes 1)\left(\psi^{\prime} \otimes 1\right)\left(1-F F^{*}\right)
$$

and

$$
\begin{aligned}
\left(\psi^{\prime} \otimes 1\right)\left(1-F F^{*}\right) & =\psi^{\prime} \otimes 1-\sum_{i} \psi^{\prime} \sigma_{i} \otimes F_{t_{i}} \sum_{j} \sigma_{j} \otimes F_{t_{j}}^{*} \\
& =\sum_{i, j} \psi^{\prime} \sigma_{i} \sigma_{j} \otimes\left(1-F_{t_{i}} F_{t_{j}}^{*}\right) .
\end{aligned}
$$

There are finitely many nonzero terms in this last double sum; each is a tensor product of a bounded-operator-valued function with a compact-operator-valued function. Since $\psi e \otimes 1$ is the tensor product of a compact-operator-valued function with a bounded-operator-valued function we see that the overall product (5.13) is compact. The various other products in (5.12) are treated similarly.

To prove part (c) it suffices to show that for every $\eta>0$, if $\varepsilon>0$ is chosen small enough then $\|[e \otimes 1, F]\|<\eta$. We compute:

$$
(e \otimes 1) F-F(e \otimes 1)=\sum_{i}\left(e \sigma_{i}-\sigma_{i} e\right) \otimes F_{t_{i}},
$$

so that

$$
\|[e \otimes 1, F]\| \leq \sup _{i}\left\|F_{i}\right\| \cdot \sum_{i}\left\|\left[e, \sigma_{i}\right]\right\| .
$$

So the result follows from Lemma 3.7.

Now let $e$ be a projection in $C\left(S^{1}\right) \otimes C^{*}(X)$. It follows from the remarks in the preceding section that, given a good family $\mathbf{F}$ and a $\varepsilon$-flat partition of unity $\sigma$ with $\varepsilon>0$ sufficiently small, we may form a $K$-theory class

$$
[\mathbf{F}, \sigma, e]=[\overline{E P}] \ominus[\overline{E Q}] \in K_{0}\left(C\left(S^{1}\right) \otimes C^{*}\left(X \times_{h}[0,1]\right)\right) .
$$

We should point out that we are not asserting that this class is independent of the choice of $\varepsilon>0$ or $\sigma$. In all that follows we shall be working with a fixed value of $\varepsilon$ which is sufficiently small for our purposes, and any fixed $\varepsilon$-flat $\sigma$.

(5.14) Lemma.

(a) Let $\tau \mapsto \mathbf{F}_{\tau}$ be a continuous map of $[0,1]$ to the set of good families. Then

$$
\left[\mathbf{F}_{0}, \sigma, e\right]=\left[\mathbf{F}_{1}, \sigma, e\right] \text {, }
$$

provided that $\sigma$ is $\varepsilon$-flat and $\varepsilon$ is sufficiently small.

(b) Let $\mathbf{F}=F_{1}, F_{2}, \ldots$ be a good family and suppose that there is a zero propagation operator $F_{0}$ on $L^{2}(\mathbb{R})$ (viewing $L^{2}(\mathbb{R})$ as a $[0,1]$-module), such that each $F_{i}$ is a compact perturbation of $F_{0}$. Suppose further that $F_{0}$ is a co-isometry, meaning that $F_{0} F_{0}^{*}=1$. Then

$$
[\mathbf{F}, \sigma, e]=\left[\left(\begin{array}{cc}
0 & 0 \\
0 & e \otimes p
\end{array}\right)\right],
$$


where $p$ is the projection operator $1-F_{0}{ }^{*} F_{0}$ on $L^{2}(\mathbb{R})$.

Proof. Part (a) is a consequence of Lemma 4.6a.

For part (b), let $\mathrm{F}_{0}=F_{0}, F_{0}, \ldots$ and apply part (a) to the family of operators

$$
\mathbf{F}_{\tau}=(1-\tau) \mathbf{F}_{0}+\tau \mathbf{F}
$$

connecting $\mathbf{F}$ to $\mathbf{F}_{0}$. We note that the idempotent associated to the constant family $F_{0}$ is

$$
P_{0}=\left(\begin{array}{cc}
1 & 0 \\
0 & 1 \otimes p
\end{array}\right),
$$

which exactly commutes with $E$. Since $P_{0} \geq Q$ we can apply Lemma $4.6 \mathrm{~b}$ to complete the proof.

\section{Proof of Theorem 5.1}

Consider the operator $-i d / d x$ on $L^{2}(\mathbb{R})$, with domain consisting of all smooth compactly supported functions. It is an essentially selfadjoint operator. Let $f: \mathbb{R} \rightarrow \mathbb{R}$ be a continuous function such that

$$
\lim _{t \rightarrow \infty} f(t)=1 \text { and } \lim _{t \rightarrow-\infty} f(t)=-1,
$$

and such that

$$
\operatorname{Supp}(\hat{f}) \subseteq[-1,1]
$$

( $\hat{f}$ denotes the Fourier transform of $f$ ). Using the functional calculus, form the bounded selfadjoint operator

$$
f(-i t d / d x): L^{2}(\mathbb{R}) \rightarrow L^{2}(\mathbb{R}) \quad(t>0) .
$$

Let $g: \mathbb{R} \rightarrow[-1,1]$ be a continuous function such that

$$
g(-s)=-1 \text { and } g(s)=1, \quad \text { for } s>0 \text { large enough. }
$$

Consider it as a bounded operator on $L^{2}(\mathbb{R})$, acting by pointwise multiplication. Form the operator

$$
F_{t}(g)=g+i\left(1-g^{2}\right)^{1 / 2} f(-i t d / d x) \quad(t>0),
$$

acting on $L^{2}(\mathbb{R})$. The following facts concerning $F_{t}(g)$ are well known.

(6.1) Lemma.

(a) The operators $F_{t}(g)$ are Fredholm, of index one, and $F_{t}(g)^{*}$ is inverse to $F_{t}(g)$, modulo compact operators.

(b) For fixed $g$ and varying $t$, the operators $F_{t}(g)$ are compact perturbations of one another.

(c) $\operatorname{Prop}\left(F_{t}(g)\right) \leq t$.

Proof. By Fourier analysis, the action of operator $f(-i t d / d x)$ (on, say, the smooth compactly supported functions), is given by convolution with the Fourier transform of $f(t \xi)$. This Fourier transform is a distribution supported in $[-t, t]$. Therefore the propagation of $f(-i t d / d x)$, and hence of $F_{t}(g)$, is no more than $t$.

It follows from this, along with our assumptions on $g$, that for large $K>0$ the operator $F_{t}(g)$ decomposes as a direct sum of three operators according to the decomposition $L^{2}(-\infty, K) \oplus L^{2}(-K, K) \oplus L^{2}(K, \infty)$ of $L^{2}(\mathbb{R})$. The first 
operator is -1 , the last is 1 , and for large enough $K$ the middle one identifies with the operator $g+i(1-g)^{1 / 2} f(-i t d / d x)$ on $L^{2}(-K, K)$, where now we take the domain of $-i d / d x$ to be the smooth functions of period $2 K$. The remaining parts of the lemma are proved by noting the corresponding facts for the middle operator. This is done by Kasparov in [5, Section 2].

For each $i=1,2, \ldots$ choose $t_{i}>0$ such that

$$
\left|t-t^{\prime}\right|<t_{i} \Rightarrow d_{h}\left((x, t),\left(x, t^{\prime}\right)\right) \leq 1, \quad \text { for all } x \in \operatorname{Supp}\left(\sigma_{i}\right)
$$

(the existence of such $t_{i}$ follows from the continuity of $h: X \times[0,1] \rightarrow Y$ ). Then $\mathbf{F}(g)=\left(F_{t_{i}}(g)\right)_{i=1}^{\infty}$ is a good family, depending continuously on $g$, and we may define an operator $F(g)$ on $L^{2}(X \times \mathbb{R})$ to be its sum with respect to a suitable $\varepsilon$-flat partition of unity; that is,

$$
F(g)=\sum_{i=1}^{\infty} \sigma_{i} \otimes F_{t_{i}}(g) .
$$

Let $e$ be a projection in $C\left(S^{1}\right) \otimes C^{*}(X)$ and choose $\varepsilon>0$ sufficiently small that we can form the $K$-theory class

$$
[\mathbf{F}(g), \sigma, e] \in K_{0}\left(C\left(S^{1}\right) \otimes C^{*}\left(X \times_{h}[0,1]\right)\right) .
$$

(6.2) Lemma. Let $g_{0}$ and $g_{1}$ be two functions satisfying the conditions imposed on $g$ above. Then

$$
\left[\mathbf{F}\left(g_{0}\right), \sigma, e\right]=\left[\mathbf{F}\left(g_{1}\right), \sigma, e\right],
$$

so long as $\varepsilon>0$ has been chosen to be small enough.

Proof. The functions $g_{\tau}=(1-\tau) g_{0}+\tau g_{1}$ all satisfy the given conditions on $g$, and the family $\mathbf{F}\left(g_{\tau}\right)$ varies continuously with $\tau$, so the lemma follows from Lemma 5.14a.

Choose $g_{0}$ so that $g_{0} \equiv 1$ on $[0, \infty)$. All the operators $F_{t}\left(g_{0}\right)$ are compact perturbations of the operator

$$
g_{0}+i\left(1-g_{0}^{2}\right)^{1 / 4} f(-i d / d x)\left(1-g_{0}^{2}\right)^{1 / 4},
$$

which decomposes as a direct sum of an index one operator on $L^{2}(-\infty, 0)$ and the identity operator on $L^{2}(0, \infty)$. The index one operator is in turn a compact perturbation of an index one co-isometry $W$ on $L^{2}(-\infty, 0)$, and so all the $F_{t}(g)$ are compact perturbations of

$$
F_{0}=W \oplus 1: L^{2}(-\infty, 0) \oplus L^{2}(0, \infty) \rightarrow L^{2}(-\infty, 0) \oplus L^{2}(0, \infty) .
$$

(6.4) Lemma. The operator $F_{0}$ in (6.3) has zero propagation (viewing $L^{2}(\mathbb{R})$ as a $[0,1]$-module).

Proof. This follows from the fact that $F_{0}$ commutes with our action of $C[0,1]$ on $L^{2}(\mathbb{R})$.

It follows from Lemma $5.14 \mathrm{~b}$ that

$$
\left[\mathbf{F}\left(g_{0}\right), \sigma, e\right]=\left[\left(\begin{array}{cc}
0 & 0 \\
0 & e \otimes p_{0}
\end{array}\right)\right],
$$


where $p_{0}=1-W_{0}^{*} W_{0}$. Since $W_{0}$ has index one, the range of the projection $p_{0}$ is a one-dimensional subspace of $L^{2}(-\infty, 0)$. So it follows from (5.3) that

$$
\left[\mathbf{F}\left(g_{0}\right), \sigma, e\right]=\left[\left(\begin{array}{cc}
0 & 0 \\
0 & e \otimes p_{0}
\end{array}\right)\right]=e_{*}^{0}[e] .
$$

We now choose $g_{1}$ so that $g_{1} \equiv-1$ on $(-\infty, 1]$. An argument similar to the one above shows that all the operators $F_{t}\left(g_{1}\right)$ are compact perturbations of a zero propagation operator

$$
F_{0}=1 \oplus W_{1}: L^{2}(-\infty, 1) \oplus L^{2}(1, \infty) \rightarrow L^{2}(-\infty, 1) \oplus L^{2}(1, \infty),
$$

where $W_{1}$ is an index-one co-isometry. Thus

$$
\left[\overline{E P\left(g_{1}\right)}\right] \ominus[\overline{E Q}]=\left[\left(\begin{array}{cc}
0 & 0 \\
0 & e \otimes p_{1}
\end{array}\right)\right]
$$

where $p_{1}=1-W_{1}^{*} W_{1}$ is the projection operator onto a one dimensional subspace of $L^{2}(1, \infty)$. It follows that

$$
\left[\overline{E P\left(g_{1}\right)}\right] \ominus[\overline{E Q}]=\left[\left(\begin{array}{cc}
0 & 0 \\
0 & e \otimes p_{1}
\end{array}\right)\right]=e_{*}^{1}[e]
$$

Putting this together with Lemma 6.2 we get

$$
e_{*}^{0}[e]=e_{*}^{1}[e] .
$$

This completes the proof of Theorem 5.1.

\section{HADAMARD MANIFOLDS}

In this section we shall exhibit some coarse homotopy equivalences.

A Hadamard manifold is a simply connected, complete Riemannian manifold with nonpositive sectional curvatures.

Let $X$ be a Hadamard manifold and fix a point 0 in $X$. Denote by $T$ the tangent space to $X$ at 0 . Since $X$ is complete, for every $v \in T$ there is a geodesic $\gamma_{v}(t)$ (defined for all $t$ ) such that

$$
\gamma_{v}(0)=0 \text { and }\left.\quad \frac{d}{d t}\right|_{t=0} \gamma_{v}(t)=v
$$

Recall that the exponential map

$$
\text { exp: } T \rightarrow X
$$

is defined by the formula

$$
\exp (v)=\gamma_{v}(1)
$$

Using the exponential map, the basic geometric properties of $X$ can be summarized as follows (see [1]).

(7.1) Proposition. Let $X$ be a Hadamard manifold and 0 any point in $X$.

(a) The exponential map exp: $T \rightarrow X$ is a diffeomorphism.

(b) If we give $T$ its euclidean metric then the inverse transformation log: $X \rightarrow$ $T$ is a nonexpansive map. That is, $d\left(\log x_{1}, \log x_{2}\right) \leq d\left(x_{1}, x_{2}\right)$, for all $x_{1}, x_{2} \in$ $X$.

Denote by $[x, y]$ the set of points on the geodesic between $x, y \in X$. 


\section{(7.2) Lemma.}

(a) (Negative Curvature Inequality.) Let $x_{0}$ and $x_{1}$ be points in $X$ and for $0 \leq t \leq 1$ let $x_{t} \in\left[x_{0}, x_{1}\right]$ be the point such that $d\left(x_{0}, x_{t}\right)=t d\left(x_{0}, x_{1}\right)$. Then for any $y \in X$,

$$
d\left(x_{t}, y\right)^{2} \leq(1-t) d\left(x_{0}, y\right)^{2}+t d\left(x_{1}, y\right)^{2}-t(1-t) d\left(x_{0}, x_{1}\right)^{2} .
$$

(b) Let $x$ and $y$ be points in $X$ with $d(0, x) \leq d(0, y)$. Denote by $x^{\prime}$ the point in $[0, y]$ such that $d\left(0, x^{\prime}\right)=d(0, x)$. Then $d\left(x, x^{\prime}\right) \leq d(x, y)$.

(c) Let $x$ and $z$ be points in $X$. For $0 \leq t \leq 1$ denote by tx the point in $[0, x]$ such that $d(0, t x)=t d(0, x)$. Define $t z$ similarly. Then

$$
d(t x, t z) \leq t d(x, z)
$$

Proof. Part (a) follows from part (b) of Proposition 7.1. The remaining parts are elementary applications of the negative curvature inequality.

We shall prove the following result:

(7.3) Theorem. The map log: $X \rightarrow T$ is a coarse homotopy equivalence.

Let $f:[0, \infty) \rightarrow[0, \infty)$ be a continuous function such that $f(0) \leq 1, f(r)$ is nonincreasing, $r f(r)$ is nondecreasing, and

$$
\lim _{r \rightarrow \infty} r f(r)=\infty \text {. }
$$

Define a map $\tilde{f}: X \rightarrow X$ by the formula

$$
\tilde{f}(x)=\exp (f(\|\log x\|) \log x),
$$

or, to use the notation of $(7.2 \mathrm{c})$,

$$
\tilde{f}(x)=f(d(0, x)) x .
$$

It is clear that $\tilde{f}$ is continuous and proper.

(7.4) Proposition. Let $f$ be as above. Then

$$
\min \{d(0, x), d(0, y)\} \leq r \quad \Rightarrow \quad d(\tilde{f}(x), \tilde{f}(y)) \leq f(r) d(x, y) .
$$

In particular,

$$
d(\tilde{f}(x), \tilde{f}(y)) \leq f(0) d(x, y) \quad \text { for all } x, y \in X
$$

Proof. Let $x, y \in X$ and suppose that $d(0, x) \leq d(0, y)$. Denote by $x^{\prime}$ the point on $[0, y]$ such that $d\left(0, x^{\prime}\right)=d(0, x)$. According to (7.2b),

$$
d\left(x, x^{\prime}\right) \leq d(x, y)
$$

Let $t_{x}=f(d(0, x))$ and $t_{y}=f(d(0, y))$. In the notation of $(7.2 \mathrm{c})$,

$$
\tilde{f}(x)=t_{x} x \text { and } \tilde{f}(y)=t_{y} y .
$$

It follows from $(7.2 \mathrm{c})$ that

$$
d\left(t_{x} x, t_{x} y\right) \leq t_{x} d(x, y)
$$

and

$$
d\left(t_{x} x, t_{x} x^{\prime}\right) \leq t_{x} d\left(x, x^{\prime}\right) \leq t_{x} d(x, y) .
$$

It follows from the hypotheses on $f$ that the point $t_{y} y$ lies between $t_{x} x^{\prime}$ and $t_{x} y$ on the line $[0, y]$ (of course it might coincide with $t_{x} x^{\prime}$ or $t_{x} y$ ). So by 
the negative curvature inequality $d\left(t_{x} x, t_{y} y\right)$ is no more than the maximum of $d\left(t_{x} x, t_{x} x^{\prime}\right)$ and $d\left(t_{x} x, t_{x} y\right)$.

(7.5) Lemma. Let $f$ be as in Proposition 7.4. Then the map $\tilde{f}: X \rightarrow X$ is coarsely homotopic to the identity map.

Proof. Given $f$ let

$$
f_{t}(r)=(1-t) r+t f(r) \quad(t \in[0,1]) .
$$

Define $h: X \times[0,1] \rightarrow X$ by $h(x, t)=\tilde{f}_{t}(x)$. This is a coarse homotopy between $\tilde{f}$ and the identity map.

Proof of Theorem 7.3. Let $a_{0}=0$, and for $n>0$ let $a_{n}$ be the maximum of the norm of the derivative of exp on the ball of radius $n+2$ about $0 \in T$. Define a piecewise linear function $F:[0, \infty) \rightarrow[0, \infty)$ by linear interpolation between the values

$$
F\left(2^{0} a_{0}\right)=0, \quad F\left(2^{1} a_{1}\right)=1, \quad F\left(2^{2} a_{2}\right)=2, \quad F\left(2^{3} a_{3}\right)=3, \ldots
$$

We obtain an increasing function, and $\lim _{r \rightarrow \infty} F(r)=\infty$. Let $f(r)=F(r) / r$. It is easily checked that $f(0)<1$ and that $f$ is nonincreasing (the powers of 2 were introduced for this purpose). Consider now the map

$$
T \stackrel{\tilde{f}}{\longrightarrow} T \stackrel{\exp }{\longrightarrow} X,
$$

from $T$ to $X$. We shall show that this is a coarse map by proving that

$$
d(v, w) \leq 1 \Rightarrow d(\exp (f(\|v\|) v), \exp (f(\|w\|) w))<1
$$

(this implies that the map (7.6) is a contraction). Suppose that $d(v, w) \leq$ 1 and that $\|v\| \leq\|w\|$. Since $T$ is a Hadamard manifold it follows from Proposition 7.4 that

$$
d(\tilde{f}(v), \tilde{f}(w)) \leq f(\|v\|) d(v, w) \leq f(\|v\|) .
$$

If we choose $n$ so that

$$
2^{n} a_{n} \leq\|v\|<2^{n+1} a_{n+1}
$$

then

$$
\|\tilde{f}(v)\|=F(\|v\|)<n,
$$

and since $\tilde{f}$ is contractive,

$$
\|\tilde{f}(w)\| \leq\|\tilde{f}(v)\|+1 \leq n+1
$$

It follows from the definition of $a_{n}$ that

$$
d(\exp \tilde{f}(v), \exp \tilde{f}(w)) \leq a_{n} d(\tilde{f}(v), \tilde{f}(w)) .
$$

Combining (7.7) with (7.8) we get

$$
d(\exp \tilde{f}(v), \exp \tilde{f}(w)) \leq a_{n} f(\|v\|) \leq a_{n} f\left(2^{n} a_{n}\right)=\frac{n-1}{2^{n-1}}<1 .
$$

This completes the proof that (7.6) is a contraction. Composing (7.6) with the map log: $X \rightarrow T$, on either side, we obtain the maps $\tilde{f}: T \rightarrow T$ and $\tilde{f}: X \rightarrow X$. 
According to Lemma 7.5 these are homotopic to the identity maps on $T$ and $X$.

(7.9) Theorem. Let $X$ be a Hadamard manifold and let $T$ be its tangent space at some point. The coarse map log: $X \rightarrow T$ induces isomorphisms

$$
\log ^{*}: H X^{*}(T) \stackrel{\cong}{\longrightarrow} H X^{*}(X)
$$

and

$$
\log _{*}: K_{*}\left(C^{*}(X)\right) \stackrel{\cong}{\longrightarrow} K_{*}\left(C^{*}(T)\right) .
$$

The assertion about coarse cohomology is already proved in [7] (using an argument which is essentially the same as our proof of Theorem 2.1). The assertion about the $K$-theory of $C^{*}(X)$ is new, and amounts to a verification of the 'coarse' Baum-Connes conjecture in the case of Hadamard manifolds. The $K$-theory of $C^{*}(T)$ may be calculated using, for example, a Mayer-Vietoris argument [3]. It is

$$
K_{j}\left(C^{*}(T)\right)= \begin{cases}\mathbb{Z} & \text { if } j \equiv \operatorname{dim}(T), \bmod 2, \\ 0 & \text { otherwise. }\end{cases}
$$

It follows from the pairings developed in [7] that the $K$-theory is generated by the index of the Dirac operator on $T$. Using Theorem 7.9 we obtain similar results about $X$ :

(7.10) Theorem. The index class of the Dirac operator on a Hadamard manifold $X$ freely generates $K_{*}\left(C^{*}(X)\right)$

The precise relation between our results and the Baum-Connes conjecture will be given elsewhere [4].

\section{REFERENCES}

1. J. Cheeger and D. Ebin, Comparison theorems in Riemannian geometry, North-Holland, Amsterdam, 1975.

2. A. Connes, Non-commutative differential geometry, Publ. Math. IHES 62 (1985), 41-144.

3. N. Higson, J. Roe and G. Yu, A coarse Mayer-Vietoris principle, Math. Proc. Cambridge Philos. Soc. 114 (1993), 85-97.

4. N. Higson and J. Roe, The coarse Baum-Connes conjecture.

5. G. Kasparov, Topological invariants of elliptic operators, I: K-homology, Math. USSR Izv. 9 (1975), 751-792.

6. G.K. Pedersen, $C^{*}$-algebras and their automorphism groups, Academic Press, London and New York, 1979.

7. J. Roe, Coarse cohomology and index theory on complete Riemannian manifolds, Mem. Amer. Math. Soc. 497 (1993).

8. J. Roe, Hyperbolic metric spaces and the exotic chomology Novikov conjecture, $K$-Theory 4 (1991), 501-512. 16802

Department of Mathematics, Penn State University, University Park, Pennsylvania

E-mail address: higson@math.psu.edu

Jesus College, OXford, OX1 3DW, England

E-mail address: jroe@jesus.ox.ac.uk 